\title{
GCU
}

Glasgow Caledonian

University

University for the Common Good

\section{Modelling the prevalence of HCV amongst people who inject drugs: an investigation into the risks associated with injecting paraphernalia sharing}

Corson, Stephen; Greenhalgh, David; Taylor, Avril; Palmateer, Norah; Goldberg, David; Hutchinson, Sharon

Published in:

Drug and Alcohol Dependence

DOI:

10.1016/j.drugalcdep.2013.05.014

Publication date:

2013

Document Version

Author accepted manuscript

Link to publication in ResearchOnline

Citation for published version (Harvard):

Corson, S, Greenhalgh, D, Taylor, A, Palmateer, N, Goldberg, D \& Hutchinson, S 2013, 'Modelling the prevalence of $\mathrm{HCV}$ amongst people who inject drugs: an investigation into the risks associated with injecting paraphernalia sharing', Drug and Alcohol Dependence, vol. 133, no. 1, pp. 172-179.

https://doi.org/10.1016/j.drugalcdep.2013.05.014

\section{General rights}

Copyright and moral rights for the publications made accessible in the public portal are retained by the authors and/or other copyright owners and it is a condition of accessing publications that users recognise and abide by the legal requirements associated with these rights.

Take down policy

If you believe that this document breaches copyright please view our takedown policy at https://edshare.gcu.ac.uk/id/eprint/5179 for details of how to contact us. 
Figure 1: Flow diagram showing the stages of HCV infection for naive PWID $(i=0)$ and experienced PWID $(i=1)$. The arrows in the diagram indicate the possible transitions for PWID between stages of HCV infection and the parameters shown are the per capita rate of flow between the stages. The rate at which PWID join and leave the population $(\mu)$ and the rate that PWID progress from the naive tier to the experienced tier $(\eta)$ are not shown.

Figure 2: Parameter plot for the high prevalence setting. This plot shows the chronic $\mathrm{HCV}$ transmission probability values required to achieve an endemic HCV prevalence of $67 \%$ (lower 95\% CI estimate, $\alpha_{y F}=0.166 \%$ ), $70 \%$ (central estimate, $\alpha_{y F}=0.193 \%$ ) and $73 \%$ (upper $95 \%$ CI estimate, $\alpha_{y F}=0.225 \%$ ).

Figure 3: Results of sensitivity analyses for the high prevalence setting. For each scenario, the figure shows the HCV transmission probability through paraphernalia sharing with chronic cases which results in a HCV prevalence of $70 \%$. The solid vertical line indicates the central estimate obtained using the baseline parameter estimates. The starred points indicate the three scenarios that lead to changes in the HCV transmission probability (through paraphernalia sharing) by a margin of more than $0.09 \%$. Note for brevity we have used ns to denote needle/syringe. 


\title{
Modelling the prevalence of HCV amongst people who inject drugs: An investigation into the risks associated with injecting paraphernalia sharing.
}

\author{
Stephen Corson ${ }^{\mathrm{a}, 1, *}$, David Greenhalgh ${ }^{\mathrm{a}}$, Avril Taylor ${ }^{\mathrm{c}}$, Norah Palmateer $^{\mathrm{b}}$, \\ David Goldberg ${ }^{\mathrm{b}}$, Sharon Hutchinson ${ }^{\mathrm{b}, \mathrm{d}}$ \\ ${ }^{a}$ Department of Mathematics and Statistics, University of Strathclyde, Livingstone Tower, 26 Richmond \\ Street, Glasgow, G1 1XH, UK. \\ ${ }^{b}$ Health Protection Scotland, Meridian Court, 5 Cadogan Street, Glasgow, G2 6QE, UK. \\ ${ }^{c}$ Institute of Applied Social and Health Research, School of Social Sciences, University of the West of \\ Scotland, Paisley, PAI 2BE, UK. \\ ${ }^{d}$ School of Health and Life Sciences, Glasgow Caledonian University, Glasgow, G4 OBA, UK.
}

\begin{abstract}
Background

In order to prevent the spread of the hepatitis $\mathrm{C}$ virus (HCV) amongst people who inject drugs (PWID), it is imperative that any injecting risk behaviour which may contribute to the transmission of disease has its role quantified. To inform public health organisations, mathematical modelling techniques were used to explore the risk of $\mathrm{HCV}$ infection through the sharing of injecting paraphernalia (including filters, cookers and water).
\end{abstract}

\section{Methods}

A mathematical model was developed for the spread of HCV based on the injecting behaviour of PWID in Scotland, with transmission occurring through the sharing of needles/syringes and other injecting paraphernalia. Numerical simulations were used to estimate the transmission probability for HCV through the sharing of injecting paraphernalia such that the modelled endemic HCV prevalence fitted with that observed amongst PWID in Scotland.

Results

The transmission probability of HCV through injecting paraphernalia was modelled to be over 8 times lower than that through needles/syringes (approximately $0.19 \%$ $0.30 \%$ and $2.5 \%$, respectively), assuming transmission occurs through a combination of at least filters and cookers. In the context of reported needle/syringe and paraphernalia sharing rates in Scotland, it is estimated that $38 \%$ and $62 \%$ of $\mathrm{HCV}$ infections

\footnotetext{
*Corresponding author

Email address: stephen. corson@strath.ac.uk (Stephen Corson)

${ }^{1}$ Tel: +44 (0)141548 3663, Fax: +44 (0)141548 3372
} 
are contributed by these practices, respectively. If needle/syringe sharing rates were to be twice those reported, the contributions would be $70 \%$ and $30 \%$, respectively.

\section{Conclusion}

Given that the sharing of injecting paraphernalia among PWID is common, HCV transmission through this route could be contributing to the growing healthcare burden associated with this chronic disease. Every effort should therefore be made to establish (a) the contribution that paraphernalia sharing is making to the spread of $\mathrm{HCV}$, and (b) the effectiveness of services providing sterile paraphernalia in preventing infection.

Keywords: hepatitis C, HCV, PWID, needle and syringe sharing, injecting paraphernalia, mathematical modelling

\section{Introduction}

The hepatitis $\mathrm{C}$ virus (HCV) is a major health concern for the 16 million (range 11-21 million) people who inject drugs (PWID) worldwide (Mathers et al., 2008). Extrapolated estimates from a systematic review of global HCV prevalence suggests that 10 million (range 6-15 million) PWID are estimated to be infected with HCV; which is 3.5 times the number of PWID who are infected with HIV (Nelson et al., 2011). The sharing of contaminated needles/syringes is considered to have the greatest role in HCV transmission (Thorpe et al., 2002; Bialek and Terrault, 2006) among PWID. However, PWID also share other injecting paraphernalia (such as cookers/spoons, filters and water) in the process of preparing drugs for injection, and they do so at greater frequency than that with which they share needles/syringes (Koester, 1996; Speed, 1998; Gaskin et al., 2000; Needle Exchange Surveillance Initiative, 2010). There is now laboratory and epidemiological evidence to suggest that the re-use of drug preparation equipment may be another source of $\mathrm{HCV}$ transmission.

In terms of laboratory studies, Crofts et al. (2000) found that it was possible to detect HCV RNA on items other than needles/syringes, including swabs (in 6 out of 9 items tested), filters (in 2/5), water samples (in 1/3) and spoons (in 1/4). Thibault et al. (2011) recently detected the presence of HCV RNA in $80 \%$ of swabs (in $4 / 5$ pools tested, involving pools of ten items) and $50 \%$ of cups (in $1 / 2$ pools tested) from suspected HCV infected PWID; HCV RNA was not detected among pools of used filters and water vials, but only a few were tested. In terms of epidemiological studies, De et al. (2008) identified ten studies that had examined HCV incidence and the sharing of injecting paraphernalia, reporting relative risks of infection associated with paraphernalia between two and six (although with wide uncertainty in these estimates). Furthermore, a meta-analysis of 8 studies, conducted by Pouget et al. (2012), recently reported that the pooled risk ratio of HCV seroconversion associated with the sharing of drug preparation equipment (excluding syringes) was similar to that for syringe sharing (2.2, 95\% CI 1.3-3.9 and 2.0, 95\% CI 1.6-2.5, respectively).

Previous modelling work in Scotland estimated that several thousand HCV infections had potentially been prevented as a consequence of reduced needle/syringe sharing rates during the late 1990s and 2000s (compared to those in the late 1980s and 
early 1990s), most likely as a result of harm reduction measures (Hutchinson et al., 2006). The prevalence of HCV among PWID in Scotland however remains high (with over half the population estimated to be infected), despite reported low levels of needle/syringe sharing (Needle Exchange Surveillance Initiative, 2008, 2010). In contrast, much higher levels of paraphernalia sharing are reported: $48 \%$ and $15 \%$ report sharing paraphernalia and needles/syringes in the past six months, respectively, (Needle Exchange Surveillance Initiative, 2010). Taking this and other available evidence into account, recently developed Scottish guidelines for services providing injecting equipment recommend that paraphernalia items - including cookers, filters and water - are made available, and that "these items be supplied in sufficient quantities to enable the use of one item each per injection" (Scottish Government, 2008, 2010).

Understanding the role that paraphernalia plays in disease transmission is fundamental to the planning and development of HCV prevention services. Rates of HCV infection following needle-stick injury among healthcare workers have demonstrated the risk of transmission through re-use of contaminated needles/syringes (Centre for Disease Control, 2001); comparable evidence does not, however, exist in relation to paraphernalia. This analysis therefore represents a first attempt to estimate the probability of HCV transmission through paraphernalia sharing.

\section{Methods}

\subsection{Model description}

We have developed a dynamic mathematical model which allows HCV to be transmitted in a PWID population through the sharing of used needles/syringes and paraphernalia items (described below); the mathematical design and equations have been described elsewhere (Corson et al., 2012a,b) and are presented in brief in the supplementary material.

We assume that the population is of size $n$, where $n$ is large and constant. PWID leave the population - either due to death or permanent cessation of injecting - at a per capita rate $\mu$ and are immediately replaced by PWID susceptible to HCV infection.

The PWID population is divided into those susceptible to HCV infection (denoted by $x$ for those previously uninfected and $x_{1}$ for those previously infected), those who have acute HCV infection ( $h_{1}$ and $h_{2}$ ), those who have developed chronic HCV infection $(y)$ and those who are immune to HCV re-infection (z) (Figure 1).

Once infected with HCV, susceptible PWID progress to an acute stage of infection (either $h_{1}$ or $h_{2}$ ). We assume that a proportion $(\delta)$ of these infected PWID progress to the acute $h_{2}$ infected class; these individuals go on to spontaneously resolve their HCV infection. The remaining proportion $(1-\delta)$ progress to the acute $h_{1}$ infected class; these individuals go on to develop chronic HCV infection. Of those PWID in the acute $h_{2}$ infected class, a proportion $(\alpha)$ go on to develop immunity to HCV re-infection and the remaining $(1-\alpha)$ become susceptible to HCV re-infection (Farci et al., 1992; Mehta et al., 2002; Micallef et al., 2007).

It has been well documented that recent initiates to injecting drug use exhibit different risk behaviour when compared to their more experienced counterparts (Cassin et al., 1998; Hahn et al., 2002; Mathei et al., 2008). Furthermore, studies report a 
higher incidence of HCV infection (Roy et al., 2007; Mehta et al., 2011) and a greater force of $\mathrm{HCV}$ infection (Sutton et al., 2006) amongst recent initiates to injecting drug use. In an attempt to capture this heterogeneity in the injecting population, the model stratified the population according to injecting career length - specifically two groups for PWID with short (naive) and long (experienced) careers. Naive PWID move at a per capita rate $\eta$ to the experienced state, and retain their HCV infection status in doing so.

\subsection{Parameter estimation}

Tables 1 and 2 summarise the baseline parameter estimates that were used in our numerical simulations.

\subsection{Acute and chronic HCV transmission probabilities through needle sharing $\left(\alpha_{h}, \alpha_{y}\right)$}

We have assumed that $\alpha_{y}=2.5 \%$ based on observed data on HCV transmission through needle-stick injury (Centre for Disease Control, 1997; Hutchinson et al., 2006). In addition, we have assumed that the infectivity of, and probability of, HCV transmission through needle sharing associated with, the acute HCV infection state $\left(\alpha_{h}\right)$ is greater than that of the chronic HCV infection state $\left(\alpha_{y}\right)$ as a result of a six to eight week period of high viraemia that follows HCV infection (Simmonds et al., 1998). In line with model fits obtained by Vickerman et al. (2007), we estimated that $\alpha_{h}=2.7 \alpha_{y}$. This implies that $\alpha_{h}=6.8 \%$.

\subsection{Proportion that develop immunity to $H C V$ re-infection $(\alpha)$}

Some, but not all, studies have shown a lower rate of HCV (re-)infection among previously infected compared to previously uninfected PWID (Micallef et al., 2007; Aitken et al., 2008; Corson et al., 2011). While this could be interpreted as immunity to $\mathrm{HCV}$ re-infection, heterogeneity in $\mathrm{HCV}$ testing intervals, small sample sizes and relatively short follow-up periods mean that it is difficult to quantify to what extent PWID who have spontaneously resolved their infection develop immunity (Corson et al., 2011; Vickerman et al., 2012). Due to the uncertainty in estimating this parameter, Vickerman et al. (2009) assumed that the proportion of PWID who become immune could range between $0 \%$ and $100 \%$. Here, we have conservatively estimated that $\alpha=0.25$.

\subsection{Duration of acute $H C V$ infection $(1 / \sigma)$}

The acute phase of infection was taken to be the first six months after initial infection (Vickerman et al., 2007, 2009). Therefore, we estimated that $(1 / \sigma)=0.5$ years or $\sigma=2$ per year.

\subsection{Proportion that resolve $H C V$ infection $(\delta)$}

We estimated that $\delta=0.26$ based on a systematic review of longitudinal studies involving 675 subjects (Micallef et al., 2006). 


\subsection{Progression rate from the naive to the experienced injecting career group ( $\eta)$}

We define naive PWID to be those who have been injecting for five years or less and experienced PWID to be those who have been injecting for more than five years, consistent with the definition used for recent onset injectors in Scottish surveillance reports (Needle Exchange Surveillance Initiative, 2010). Hence, $1 / \eta=5$ years.

\subsection{Proportion of PWID that are naive and experienced $\left(\pi^{0}, \pi^{1}\right)$}

We assumed that $25 \%$ of the PWID population were naive and the remaining $75 \%$ are experienced. This is consistent with current PWID in Glasgow, surveyed at services providing injecting equipment in 2008-2009, where 23\% (164/704) had commenced injecting in the past five years (Needle Exchange Surveillance Initiative, 2010). In addition, the size of the Glasgow PWID population has been relatively constant over a number of years so we assume that our estimates for $\pi^{0}$ and $\pi^{1}$ are the equilibrium values for these parameters. That is, $\pi^{0}=\pi^{0 *}=0.25$ and $\pi^{1}=\pi^{1 *}=0.75$.

\subsection{Average length of injecting career $(1 / \mu)$}

At equilibrium, the fraction of naive PWID in the population is given by $\mu /(\mu+$ $\eta)$. Re-arranging this for $\mu$ and using our estimates for $\pi^{0 *}$ and $\eta$ we see that $\mu=$ 0.06667 per PWID per year. This implies that the average length of injecting career is approximately 15 years.

\subsection{Probability of successful needle/syringe cleaning $(\phi)$}

We used data from a survey of PWID conducted in Glasgow during 1990-93 to estimate $\phi$ (Hutchinson et al., 2000). Among the PWID who had reported injecting with a used needle/syringe in the past six months, the majority $(91 \%, 620 / 679)$ reported they always cleaned their needles before use ( 155 bleach, 15 alcohol, 105 boiling water, 345 other); 24 mostly cleaned their needles ( 5 bleach, 3 boiling water, 16 other), 8 cleaned about half the time ( 2 bleach, 6 other), 11 cleaned occasionally ( 1 bleach, 10 other), 14 never cleaned and the remaining 2 said that they did not know.

We assumed that cleaning with bleach is $99.9 \%$ effective and alcohol is $85 \%$ effective at disinfecting needles/syringes infected with HCV (National Treatment Agency, 2009); other methods were assumed to be completely ineffective. We also assumed that those individuals who mostly clean used needles prior to use will do this on average $87 \%$ of the time, those who clean about half the time will do so $50 \%$ of the time, and those who occasionally clean will do so on average $13 \%$ of the time. We assumed that the few individuals who reported that they did not know were completely ineffective at cleaning their needles before use. Applying these assumptions, we estimate that 173 of $679(25.5 \%)$ PWID, who reported sharing needles/syringes, would have cleaned their needles successfully the last time they injected, providing an estimate for $\phi$ of 0.255 . 


\subsection{Number of needle/syringe sharing events $\left(\lambda_{0}, \lambda_{1}\right)$}

Estimates for $\lambda_{0}$ and $\lambda_{1}$ were obtained from a survey of 704 current PWID (those who reported injecting drugs in the six month period prior to interview) in Glasgow during 2008-2009 (Needle Exchange Surveillance Initiative, 2010). We identified 162 naive and 535 experienced PWID who reported (a) the number of months spent injecting, and (b) the frequency at which they shared needles/syringes (defined as injecting with a needle/syringe that had already been used by someone else) in the one month prior to interview. Of these, $10(6 \%)$ naive and 47 (9\%) experienced PWID reported that they had shared needles/syringes at least once in the month prior to interview; these 10 naive PWID reported sharing a total of 74 times (7.4 times on average each) per month and the 47 experienced PWID reported sharing a total of 469 times (9.98 times on average each) per month. Naive PWID reported injecting for 8 months of the year, thus we estimated that there were $592(74 \times 8)$ needle/syringe sharing events per year among the 162 naive PWID. Similarly, experienced PWID reported injecting for 8.8 months of the year, and so there were an estimated $4,127(469 \times 8.8)$ needle/syringe sharing events per year among the 535 experienced PWID. We therefore assumed that the number of times in a year that a PWID shared needles/syringes was $3.7(592 / 162)$ for all those naive, and $7.7(4,127 / 535)$ for all those experienced.

\subsection{Number of paraphernalia sharing events $\left(\lambda_{0 F}, \lambda_{1 F}\right)$}

From a survey of 704 current PWID in Glasgow during 2008-2009 (Needle Exchange Surveillance Initiative, 2010), we identified 160 naive and 540 experienced PWID of whom 61 (38\%) naive and 183 (25\%) experienced PWID reported that they had shared injecting paraphernalia items at least once in the one month period prior to interview. Since recent surveys do not quantify the frequency of paraphernalia sharing in the period prior to interview, we assumed that the distribution of sharing events amongst those who reported paraphernalia sharing in 2008-2009 was similar to that obtained from a survey of 564 current PWID who reported sharing injecting paraphernalia in Glasgow in 2004. Allowing for months out of injecting (see above), we estimated that there were 14,924 paraphernalia (3,936 filter, 5,436 cooker and 5,552 water) sharing events per year for the 160 naive PWID and 62,180 paraphernalia $(19,140$ filter, 21,296 cooker, and 21,744 water) sharing events per year for the 540 experienced PWID. Therefore, the average paraphernalia sharing rate for naive PWID $\left(\lambda_{O F}\right)$ was 93 sharing events per year and the paraphernalia sharing rate for experienced PWID $\left(\lambda_{1 F}\right)$ was 115 sharing events per year.

\subsection{Fraction of needles/syringes and injecting paraphernalia sharing events that oc-} cur within injecting career groups $\left(s_{00}, s_{11}, \bar{s}_{00}, \bar{s}_{11}\right)$ and across injecting career groups $\left(s_{01}, s_{10}, \bar{s}_{01}, \bar{s}_{10}\right)$

No data sources were available to estimate the proportion of sharing that goes on within injecting career groups (between naive PWID or between experienced PWID) or across groups (between naive PWID and experienced PWID). We assumed that PWID will tend to borrow equipment (needles/syringes or injecting paraphernalia) from PWID in the same injecting career group. Hence we estimated that $s_{00}=\bar{s}_{00}=$ $s_{11}=\bar{s}_{11}=0.75$ and $s_{01}=\bar{s}_{01}=s_{10}=\bar{s}_{10}=0.25$. 


\subsection{Simulations}

Our aim was to determine the probability of HCV transmission through the sharing of injecting paraphernalia (including filters, cookers and water).

Using the differential equation solver ode 45 in MATLAB (2011) and the baseline set of parameter estimates given in Tables 1 and 2, we modelled the prevalence of HCV amongst PWID in Glasgow (a high prevalence setting). We report the transmission probabilities that generated an endemic HCV prevalence consistent with that observed from PWID surveyed in Glasgow during 2008-2009, in that the modelled prevalence needed to lie within the $95 \% \mathrm{CI}$ of the observed $\mathrm{HCV}$ antibody prevalence, i.e. between $67 \%$ and $73 \%$, (Needle Exchange Surveillance Initiative, 2010).

In the baseline model, we assume that the probability of transmission relating to the sharing of injecting paraphernalia with an individual with acute HCV infection was 2.7 times greater than that relating to sharing with an individual with chronic $\mathrm{HCV}$, as was assumed for the transmission probabilities associated with the sharing of needles/syringes. Hence, $\alpha_{h F}=2.7 \alpha_{y F}$ and so the focus is on estimating the transmission probability relating to the sharing of paraphernalia with a chronically infected individual. Note that the estimated transmission probabilities are for the collective sharing of filters, cookers and water, not for each item individually.

To validate the model, we repeated our simulations in a low prevalence setting (Table 1 in the supplementary material provides a summary of the parameter estimates specific to the low prevalence setting). In this instance, we modelled the prevalence of HCV amongst PWID in Lothian (the healthboard relating to Edinburgh city in Scotland) and we report the transmission probabilities that generated an endemic HCV prevalence consistent with that observed from PWID surveyed in Lothian during 20082009 (31\%, 95\% CI 25\%-36\%) (Needle Exchange Surveillance Initiative, 2010).

\subsection{Sensitivity analysis}

A sensitivity analysis was conducted for the high prevalence setting to investigate how the central estimate for the transmission probability relating to the sharing of paraphernalia with a chronically infected individual was affected by the uncertainty in different parameter estimates.

Several one factor at a time sensitivity analyses were performed on the following parameters: (i) the probability of successful needle/syringe cleaning (reduced to 0 and increased to 0.5), (ii) the proportion that develop immunity to HCV re-infection (reduced to 0 and increased to 0.5 ), (iii) the acute $\mathrm{HCV}$ transmission probability through needle sharing (reduced to $2.5 \%$ which assumes that there is no period of high viraemia following $\mathrm{HCV}$ infection), (iv) the chronic $\mathrm{HCV}$ transmission probability through needle/syringe sharing (reduced to $1.5 \%$ and increased to 5\% which are the lower and upper estimates obtained from model fits to data from Rawalpindi, Pakistan (Vickerman et al., 2009)), (v) the proportion of PWID that are naive (increased to 0.3333), (vi) the number of paraphernalia sharing events (reduced to 59 events per year for naive and 75 events per year for experienced PWID assuming HCV transmission does not occur through the sharing of water), (vii) the number of needle/syringe sharing events (doubled to 7.4 events per year for naive and 15.4 events per year for experienced PWID and tripled to 11.1 events per year for naive and 23.1 events per year for experienced PWID 
to account for under-reporting), (viii) the swapping of the baseline needle/syringe (and paraphernalia) sharing rates for naive and experienced PWID, and (ix) the fraction of needle/syringe sharing events that occur within experience groups (reduced to 0.25 and increased to 1). It is worth noting that (a) the proportional relationship between acute and chronic HCV infection is maintained when the transmission probability of chronic HCV through needles/syringes is reduced to $1.5 \%$ and increased to $5 \%$, and (b) changes to these parameters affected the baseline estimates of other parameters (e.g. the fraction of needle/syringe sharing events that occur across injecting career groups).

\section{Results}

\subsection{Determining the probability of HCV transmission through the sharing of injecting paraphernalia (including filters, cookers and water)}

Figure 2 shows a plot of HCV prevalence (defined as those testing HCV antibody positive, which is given by $\pi_{x_{1}}^{0}+\pi_{h_{1}}^{0}+\pi_{h_{2}}^{0}+\pi_{y}^{0}+\pi_{z}^{0}+\pi_{x_{1}}^{1}+\pi_{h_{1}}^{1}+\pi_{h_{2}}^{1}+\pi_{y}^{1}+\pi_{z}^{1}$ in our model) as a function of $\alpha_{y F}$, the transmission probability of chronic HCV through paraphernalia sharing.

For the high prevalence setting (Glasgow), we estimated that in order to achieve an endemic HCV prevalence between $67 \%$ and $73 \%$, we require the HCV transmission probability through sharing paraphernalia with a chronically infected individual to be between $0.166 \%$ and $0.225 \%$, with a central estimate of $0.193 \%$, approximately 13 times lower than the transmission probability associated with needle/syringe sharing $\left(\alpha_{y}=2.5 \%\right)$. These estimates imply that the HCV transmission probability through the sharing of paraphernalia with an acutely infected individual is between $0.448 \%$ and $0.608 \%$.

For the low prevalence setting, we estimated that in order to achieve an endemic $\mathrm{HCV}$ prevalence of $31 \%$ (and between $25 \%$ and $36 \%$ ) we require the HCV transmission probability through sharing paraphernalia with a chronically infected individual to be $0.170 \%$ (and between $0.157 \%$ and $0.183 \%$ ), approximately 15 times lower than the transmission probability associated with needle/syringe sharing. These estimates imply that the HCV transmission probability through sharing paraphernalia with an acutely infected individual is between $0.424 \%$ and $0.494 \%$.

\subsection{Sensitivity analysis}

The results of our sensitivity analyses are presented in Figure 3. Three scenarios lead to changes in the HCV transmission probability (through paraphernalia sharing) by a margin of more than $0.09 \%$. First, reducing the number of paraphernalia sharing events per year by excluding the water sharing events results in an increase in our estimate for the transmission probability of chronic HCV through paraphernalia sharing (from $0.193 \%$ to $0.301 \%$ ). Second, doubling to tripling the number of needle/syringe sharing events results in our estimate decreasing from $0.193 \%$ to $0.10 \%$ and $0.008 \%$, respectively. Third, increasing the transmission probability of chronic HCV through needles/syringes to its upper estimate of $5 \%$ results in our estimate decreasing from $0.193 \%$ to $0.10 \%$. 


\subsection{Proportion of infections attributable to paraphernalia sharing}

To estimate the proportion of infections attributable to paraphernalia sharing, we used the central estimate for the transmission probability relating to the sharing of paraphernalia with a chronically infected individual to calculate the equilibrium force of infection (given by (13) in the supplementary material).

Several scenarios were considered: relating to the baseline parameters for the high and low prevalence settings (with $\alpha_{y f}=0.193 \%$, and $0.170 \%$, respectively); and in the high prevalence setting, reducing the number of paraphernalia sharing events per year by excluding the water sharing events (with $\alpha_{y f}=0.301 \%$ ), and doubling and tripling the needle/syringe sharing rates (with $\alpha_{y f}=0.10 \%$ and $0.008 \%$, respectively).

Our results showed that approximately $62 \%$ of infections in the high prevalence setting and $73 \%$ of infections in the low prevalence setting can be attributed to paraphernalia sharing. Furthermore, in the high prevalence setting, excluding water sharing events has little impact on the proportion of infections attributable to paraphernalia sharing (63\% compared to $62 \%$ ). However, doubling the needle/syringe sharing rates halves the proportion of infections attributable to paraphernalia sharing (30\% compared to $62 \%$ ), while tripling the needle/syringe sharing rates reduces this proportion even further ( $2 \%$ compared to $62 \%$ ).

\section{Conclusions and discussion}

Laboratory studies and epidemiological surveys have suggested that the sharing of injecting paraphernalia (such as cookers, filters and water) may contribute to the spread of HCV amongst PWID (Crofts et al., 2000; De et al., 2008; Pouget et al., 2012). PWID in Scotland have reported much higher levels of paraphernalia sharing than needle/syringe sharing and it is possible that the former may explain the high $\mathrm{HCV}$ prevalence that is observed in this population (Needle Exchange Surveillance Initiative, 2010). With this in mind, recently developed Scottish guidelines for injecting equipment provision have recommended an increase in the provision of paraphernalia items (cookers, filters and water) so that every injector has access to a sterile set of equipment for each injection (Scottish Government, 2010). Given that the resources required to meet the guidelines are likely to be substantial, it is important to quantify the role that the sharing of these items plays in disease transmission.

Here we have developed a dynamic mathematical model that allowed HCV transmission to occur through the sharing of needles/syringes and other injecting paraphernalia (defined as cookers, filters and water). Using parameter estimates obtained from surveys of Scottish PWID, we simulated the behaviour of the model and estimated the transmission probability of HCV through the sharing of injecting paraphernalia. Our numerical simulations implied that, provided that the reported needle/syringe sharing rates are accurate, the probability of $\mathrm{HCV}$ transmission through sharing paraphernalia (either cookers, filters or water) with a chronically infected individual was approximately $0.19 \%$; this estimate was approximately 13 times lower than the transmission probability associated with needle/syringe sharing (2.5\%). This result was corroborated by numerical simulations performed with data on PWID from Lothian (a low 
prevalence setting) which generated a transmission probability estimate of approximately $0.17 \%$. Furthermore, we estimated that approximately $62 \%$ and $73 \%$ of infections in the high and low prevalence settings, respectively, were attributable to paraphernalia sharing (assuming the baseline needle/syringe sharing rates for both settings are correct). A recent analysis of Scottish survey data by Palmateer et al. (2012) found that amongst PWID who did not report needle/syringe sharing, the sharing of filters and spoons was significantly associated with recent HCV infection, but the sharing of water was not. Therefore, we reduced the number of paraphernalia sharing events by excluding water sharing and found that this increased our estimate from $0.19 \%$ to $0.30 \%$ per paraphernalia sharing event with a chronically infected individual; the latter estimate was 8 times lower than the transmission probability associated with needle/syringe sharing.

These results suggest that the sharing of injecting paraphernalia could potentially contribute to the spread of HCV amongst PWID. These results are supported by Mathei et al. (2006) whose simulation study considered HCV transmission through paraphernalia sharing and examined how sharing behaviours affect the force of infection. Their results suggested that PWID who reported the sharing of injecting paraphernalia experienced a greater force of infection and had a higher long term HCV prevalence than those who did not report the sharing of injecting paraphernalia.

A recent meta-analysis by Pouget et al. (2012) suggested that the risk of HCV infection through shared drug preparation equipment was similar to that of shared syringes. This is based on the finding that the pooled risk ratio of HCV seroconversion was 1.94 (95\% CI 1.53-2.46) for those who had shared a syringe and 2.24 (95\% CI 1.28-3.93) for those who had shared drug preparation equipment. The authors did not present information on the number of shared injection episodes in their review, most likely because this was also not available in the primary articles, and therefore did not examine differences in risk of $\mathrm{HCV}$ according to the number of events involving shared needles/syringes and other paraphernalia. In our model, we were able to build in the number of sharing events for each item of injection equipment; in doing so, we highlight that the number of paraphernalia sharing events is far in excess of that for needles/syringes (that is, 17 times more paraphernalia, compared to needle/syringe, sharing events per PWID per year in Glasgow). Allowing for these data, we illustrate that the transmission risk associated with each paraphernalia sharing event is therefore many times lower than that of a needle/syringe sharing event.

To estimate the number of needle/syringe sharing events for naive and experienced PWID in Glasgow, we used needle/syringe sharing frequency data extracted from the 2008-2009 survey of PWID in Glasgow. The estimates obtained from this data (3.7 and 7.7 for naive and experienced PWID, respectively) were found to be: (a) consistent with the needle/syringe sharing frequency reported by Glasgow PWID in surveys since conducted in 2010 and 2011-2012, and (b) in line with low levels of needle/syringe sharing in other countries that have implemented substantial injecting equipment programmes such as England and Australia (The Kirby Institute, 2011; Health Protection Agency, 2012). Nevertheless, it is entirely possible that PWID under-report their needle/syringe sharing risk behaviour. Sensitivity analyses, which assumed that needle/syringe sharing rates were double that reported in Glasgow during 
2008-2009, showed that the model required a much smaller estimate for the probability of $\mathrm{HCV}$ transmission through paraphernalia sharing to produce prevalence estimates that fitted with that observed amongst Glasgow PWID in 2008-2009 (0.1\% compared to $0.19 \%$ ). This potential bias would mean that we have under-estimated the number of needle/syringe sharing events and over-estimated the risk of HCV infection through paraphernalia sharing. Given that our estimates are sensitive to the number of needle/syringe sharing events, it is important that we understand to what extent PWID under-report their needle/syringe sharing so that we can gain a more accurate understanding of the potential risks associated with the sharing of injecting paraphernalia.

It is worth noting that our baseline model applied a higher number of sharing events (needle/syringe and paraphernalia) to experienced PWID than naive PWID. Although this may seem counter intuitive, our sensitivity analyses showed that this discrepancy did not invalidate our results: swapping the baseline naive and experienced needle/syringe (and paraphernalia) sharing rates reduced our estimate by $0.02 \%$ to $0.17 \%$.

In our analysis we have assumed that the transmission probability of chronic $\mathrm{HCV}$ through needles/syringes $\left(\alpha_{y}\right)$ is $2.5 \%$ (Centre for Disease Control, 1997; Hutchinson et al., 2006), while more generally, estimates obtained from model fits to data from Pakistan suggest that $\alpha_{y}$ lies between 1.5\% and 5\% (Vickerman et al., 2009). Our sensitivity analyses showed that an increase in $\alpha$ yould almost halve the risk of infection associated with paraphernalia sharing; the probability of HCV transmission through sharing paraphernalia with a chronically infected individual decreased approximately $0.19 \%$ to $0.1 \%$ as $\alpha_{y}$ was increased from $2.5 \%$ to $5 \%$. Quantifying the risks associated with needle/syringe sharing is thus essential to fully appreciate the risks associated with paraphernalia sharing.

Although this analysis examines the risks associated with the sharing of filters, cookers and water, there is some uncertainty as to which items of injecting paraphernalia should be considered as potential HCV transmission sources. For example, Crofts et al. (2000) found that 40\% (2/5) of filters tested had detectable levels of HCV RNA which suggests that filters could be a viable source of HCV infection. In contrast Thibault et al. (2011) did not detect HCV RNA in the pool of ten filters collected from PWID who reported that they were infected with HCV. Although the authors mention that no firm conclusions can be drawn on the potential risk of contamination though the sharing of filters, this discrepancy highlights the need for more studies designed to investigate the risks associated with shared paraphernalia.

As with all modelling studies there are a number of limitations. The deterministic nature of our model does not allow us to incorporate the random variability in needle/syringe sharing rates and paraphernalia sharing rates. That said, we do allow for a level of heterogeneity in these sharing rates by modelling the spread of HCV infection amongst naive and experienced PWID, each with different risk behaviours. It is reasonable to consider that the transmission probability of $\mathrm{HCV}$ through one paraphernalia item (e.g. filters) will be different from the other items (e.g. cookers and water). Our simulations were unable to generate separate transmission probabilities for filters, cookers and water. Therefore, we are unable to determine whether one particular item of equipment poses a greater risk than the others. When calculating the parameter esti- 
mate for $\phi$ (the probability of cleaning needles/syringes) we found that recent surveys of PWID in Glasgow did not quantify the frequency of needle/syringe cleaning in the period prior to interview. The use of cleaning data from the early 1990s may result in an under-estimation of the level of preventative cleaning that is ongoing within the Glasgow PWID population which would in turn lead to our model under-estimating the risks associated with paraphernalia sharing. Furthermore, we conservatively assumed that only two needle/cleaning methods (bleach and alcohol) were effective at sterilising HCV infected needles/syringes. Studies by Abdala et al. $(2001,2004)$ have suggested that several methods may have some efficacy when it comes to inactivating blood-borne infections and thus our estimate for $\phi$ may be further under-estimated. However, sensitivity analyses showed that our model was not overly sensitive to this parameter. Finally, our modelling work does not consider non-injecting transmission routes for $\mathrm{HCV}$ (such as tattooing and sexual transmission). If these transmission routes were to be incorporated, it is possible that our estimate for injecting paraphernalia transmission would be somewhat reduced.

In conclusion, our results suggest that the sharing of injecting paraphernalia (such as cookers, water and filters) could be contributing to the growing healthcare burden associated with HCV. Given that PWID are reporting that they share injecting paraphernalia at a much greater frequency than with which they share needles/syringes, it is imperative that every effort be made to establish (a) the contribution that paraphernalia sharing is making to the ongoing spread of $\mathrm{HCV}$, and (b) the effectiveness of services providing sterile paraphernalia in preventing infection. It may be difficult to determine from observational studies the risk of HCV transmission for each paraphernalia item, given the extent to which these behaviours co-exist. Nevertheless, a natural experiment is underway in Scotland - as a result of extra investment from Government (Scottish Government, 2008) and new national guidelines (Scottish Government, 2010) - which will inform on whether the increased provision of sterile paraphernalia (especially filters and cookers) is associated with a reduced risk of HCV transmission among PWID. 


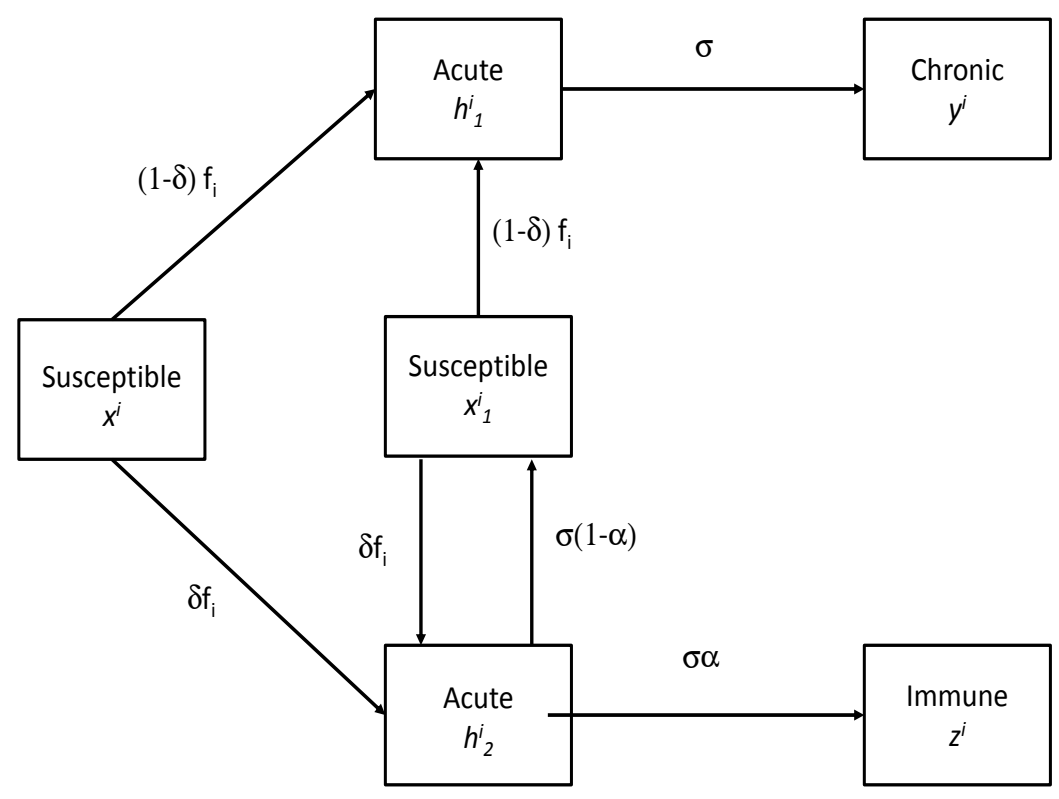

Figure 1:

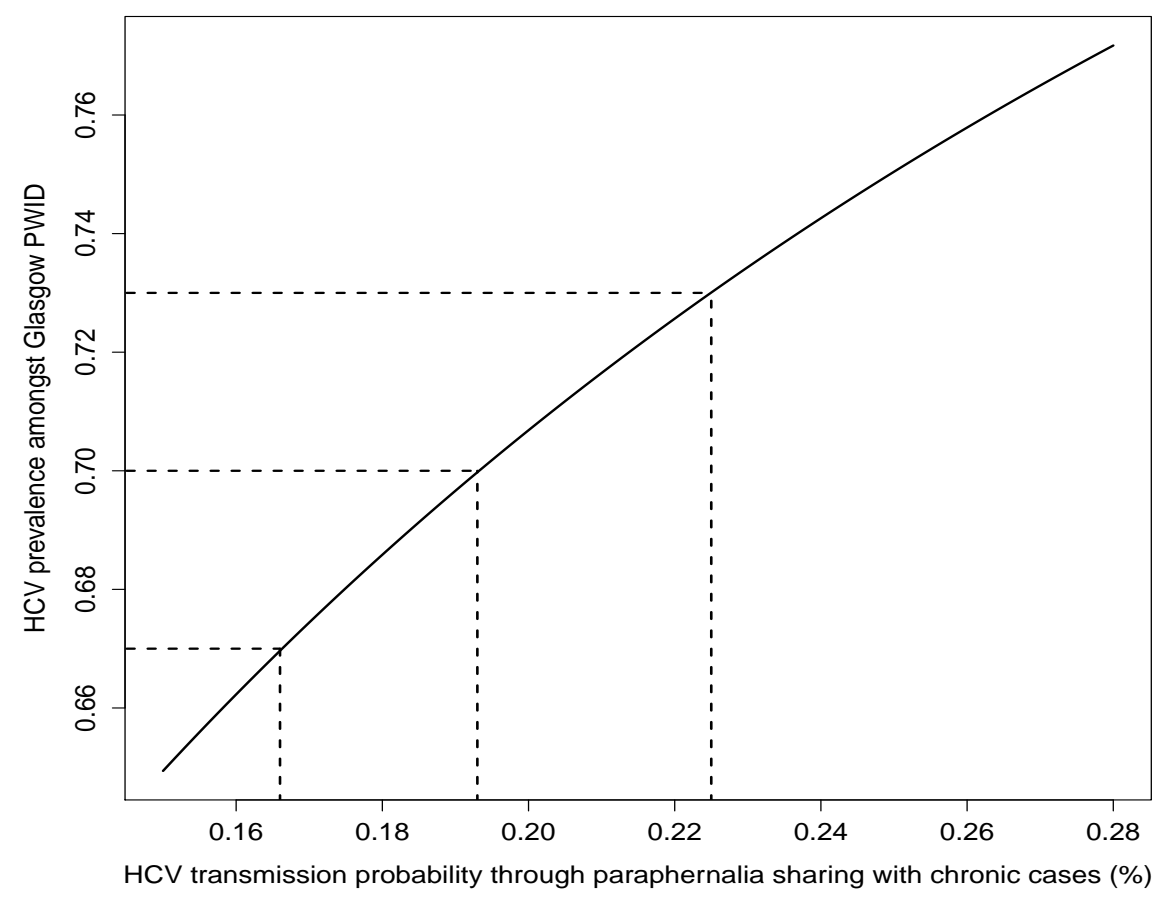

Figure 2: 
Probability of successful needle cleaning reduced to 0

Probability of successful needle cleaning increased to 0.5

Immunity to $\mathrm{HCV}$ re-infection reduced to 0

Immunity to HCV re-infection increased to 0.5

No increased viraemia following initial infection

Reduced transmission probability of chronic HCV (to 1.5\%) through ns sharing Increased transmission probability of chronic HCV (to 5\%) through ns sharing Proportion of naïve PWID increased to 0.3333

Number of paraphernalia sharing events reduced to exclude water sharing events Doubling the number of ns sharing events

Tripling the number of ns sharing events

Swapping the naive and experienced ns sharing rates

Fraction of ns sharing events that occur between naïve PWID reduced to 0.25

Fraction of ns sharing events that occur between naive PWID increased to 1

Fraction of ns sharing events that occur between experienced PWID reduced to 0.25

Fraction of ns sharing events that occur between experienced PWID increased to 1

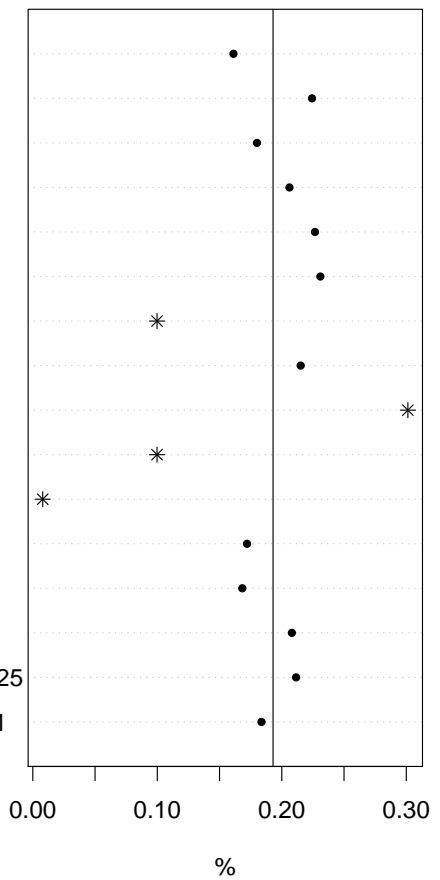

Figure 3: 


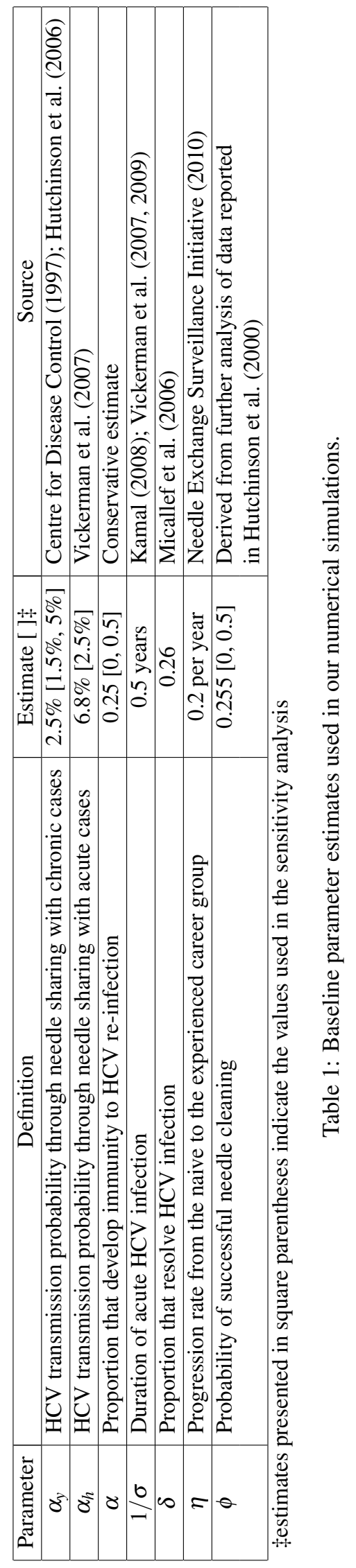




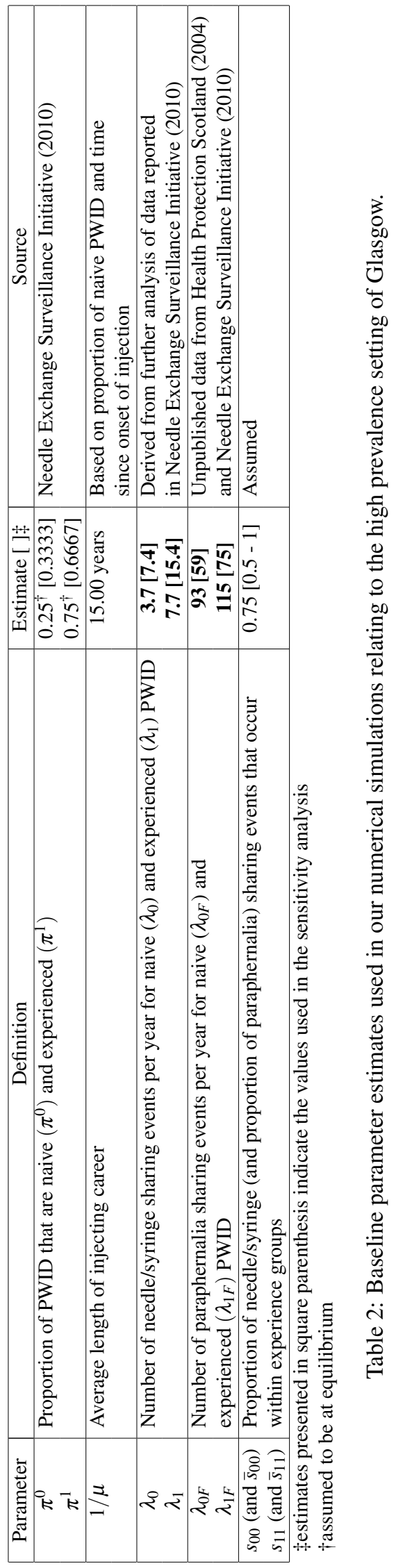




\section{References}

Abdala, N., Crowe, M., Tolstov, Y., Heimer, R., 2004. Survival of Human Immunodeficency Virus type 1 after rinsing injection syringes with different cleaning solutions. Subst. Use Misuse 39, 581-600.

Abdala, N., Gleghorn, A.A., Carney, J.M., Heimer, R., 2001. Can HIV-1 contaminated syringes be disinfected? Implications for transmission among injection drug users. J. Acquir. Immune Defic. Syndr. 28, 487-494.

Aitken, C.K., Lewis, J., Tracy, S.L., Spelman, T., Bharadwaj, M., Bowden, D.S., Drummer, H., Hellard, M.E., 2008. High incidence of hepatitis C virus reinfection in a cohort of injecting drug users. Hepatology 48, 1749-1752.

Bialek, S., Terrault, N., 2006. The changing epidemiology and natural history of hepatitis $\mathrm{C}$ virus infection. Clin. Liver Dis. 10, 697-715.

Cassin, S., Geoghegan, T., Cox, G., 1998. Young injectors: A comparative analysis of risk behaviour. Ir. J. Med. Sci. 167, 234-237.

Centre for Disease Control, 1997. Recommendations for follow-up of health-care workers after occupational exposure to hepatitis $C$ virus. Technical Report 46 . Centre for Disease Control. Altanta, Georgia, USA.

Centre for Disease Control, 2001. Updated US public health service guidelines for the management of occupational exposures to $\mathrm{HBV}, \mathrm{HCV}$ and $\mathrm{HIV}$ and recommendations for post exposure prophylaxis. Technical Report 50. Centre for Disease Control. Altanta, Georgia, USA.

Corson, S., Greenhalgh, D., Hutchinson, S., 2012a. Mathematically modelling the spread of hepatitis C in injecting drug users. Math. Med. Biol. 29, 205-230.

Corson, S., Greenhalgh, D., Hutchinson, S.J., 2012b. A time since onset of injection model for hepatitis $\mathrm{C}$ spread amongst injecting drug users. J. Math. Biol. 66, 935978.

Corson, S., Greenhalgh, D., Palmateer, N., Weir, A., Hutchinson, S., 2011. Risk of hepatitis $\mathrm{C}$ virus re-infection following spontaneous viral clearance in injecting drug users: A systematic review. Int. J. Drug Policy 22, 102 - 108.

Crofts, N., Caruana, C., Bowden, S., Kerger, M., 2000. Minimising harm from hepatitis $\mathrm{C}$ virus needs better strategies. BMJ 321, 899.

De, P., Roy, E., Boivin, J.F., Cox, J., Morissette, C., 2008. Risk of hepatitis C virus transmission through drug preparation equipment: A systematic and methodological review. J. Viral Hepat. 15, 279-292. 
Farci, P., Alter, H.J., Govindarajan, S., Wong, D.C., Engle, R., Lesniewski, R.R., Mushahwar, I.K., Desai, S.M., Miller, R.H., Ogata, N., Purcell, R.H., 1992. Lack of protective immunity against reinfection with hepatitis $\mathrm{C}$ virus. Science 258, 135140 .

Gaskin, S., Brazil, C., Pickering, D., 2000. The sharing of injecting paraphernalia by intravenous drug users (IDUs) within a Worcestershire cohort, with specific reference to water and filters. Int. J. Drug Policy 11, 423-435.

Hahn, J.A., Page-Shafer, K., Lum, P.J., Bourgois, P., Stein, E., Evans, J.L., Busch, M.P., Tobler, L.H., Phelps, B., Moss, A.R., 2002. Hepatitis C virus seroconversion among young injection drug users: Relationships and risks. J. Infec. Dis. 186, 15581564. http://jid.oxfordjournals.org/content/186/11/1558.full.pdf+ html.

Health Protection Agency, 2012. Shooting up. Infections among injecting drug users in the United Kingdom 2012. Technical Report. Health Protection Agency Centre for Infections, Colindale, London, UK.

Health Protection Scotland, 2004. Health Protection Scotland survey of Glasgow IDUs in 2004. Health Protection Scotland, Glasgow, UK.

Hutchinson, S., Bird, S., Taylor, A., Goldberg, D., 2006. Modelling the spread of hepatitis $\mathrm{C}$ virus infection among injecting drug users in Glasgow: Implications for prevention. Int. J. Drug Policy 17, 211-221.

Hutchinson, S., Taylor, A., Goldberg, D., Gruer, L., 2000. Factors associated with injecting risk behaviour among serial community-wide samples of injecting drug users in Glasgow 1990 to 1994: Implications for control and prevention of bloodborne viruses. Addiction 95, 931-940.

Kamal, S.M., 2008. Acute hepatitis C: A systematic review. Am. J. Gastroenterol. $103,1283-1297$.

Koester, S., 1996. The process of drug injection. Applying ethnography to the study of HIV risk among IDUs., in: Hartnol, R., Rhodes, T. (Eds.), AIDS, drugs and prevention, Routledge, London, UK. pp. 133-149.

Mathei, C., van Dooreen, S., Lemey, P., van Damme, P., Buntinx, F., Vandamme, A.M., 2008. The epidemic history of hepatitis $\mathrm{C}$ among injecting drug users in Flanders, Belgium. J. Viral Hepat. 15, 399-408.

Mathei, C., Shkedy, Z., Denis, B., Kabali, C., Aerts, M., Molenderghs, G., van Damme, P., Buntinx, F., 2006. Evidence for a substantial role of sharing of injecting paraphernalia other than syringes/needles to the spread of hepatitis $\mathrm{C}$ among injecting drug users. J. Viral Hepat. 13, 567-570. 
Mathers, B.M., Degenhardt, L., Phillips, B., Wiessing, L., Hickman, M., Strathdee, S.A., Wodak, A., Panda, S., Tyndall, M., Toufik, A., Mattick, R.P., 2008. Global epidemiology of injecting drug use and HIV among people who inject drugs: A systematic review. The Lancet 372, 1733-1745.

MATLAB, 2011. version 7.13.0.564 (R2011b). The MathWorks Inc., Natick, Massachusetts.

Mehta, S.H., Astemborski, J., Kirk, G.D., Strathdee, S.A., Nelson, K.E., Valhow, D., Thomas, D.L., 2011. Changes in blood-borne infection risk among injection drug users. J. Infect. Dis. 203, 587-594.

Mehta, S.H., Cox, A., Hoovere, D.R., Wang, X., Mao, Q., Ray, S., Strathdee, S.A., Vlahov, D., Thomas, D., 2002. Protection against persistence of hepatitis C. The Lancet 359, 1478-1483.

Micallef, J.M., Kaldor, J.M., Dore, G.J., 2006. Spontaneous viral clearance following acute hepatitis $\mathrm{C}$ infection: A systematic review of longitudinal studies. J. Viral Hepat. 13, 34-41.

Micallef, J.M., Macdonald, V., Jauncey, M., Amin, J., Rawlinson, W., van Beek, I., 2007. High incidence of hepatitis $C$ virus infection within a cohort of injecting drug users. J. Viral Hepat. 14, 413-418.

National Treatment Agency, 2009. Does syringe cleaning work? http: //www.harmreductionworks.org.uk/2\_films/newlinedoes\_cleaning \ _syringes\_work.html. Accessed (2010).

Needle Exchange Surveillance Initiative, 2008. The Needle Exchange Surveillance Initiative (NESI): Prevalence of HCV, HIV and injecting risk behaviours among injecting drug users attending needle exchanges in Scotland, 2007. Technical Report. Health Protection Scotland and the University of the West of Scotland.

Needle Exchange Surveillance Initiative, 2010. The Needle Exchange Surveillance Initiative (NESI): Prevalence of HCV and injecting risk behaviours among injecting drug users attending needle exchanges in Scotland, 2008/2009. Technical Report. University of the West of Scotland and Health Protection Scotland and West of Scotland Specialist Virology Centre, Paisley, UK.

Nelson, P.K., Mathers, B.M., Cowie, B., Hagan, H., des Jarlais, D., Horyniak, D., Degenhardt, L., 2011. Global epidemiology of hepatitis B and hepatitis C in people who inject drugs: Results of systematic reviews. The Lancet 378, 571-583.

Palmateer, N., Hutchinson, S.J., McAllister, G., Munro, A., Cameron, S., Goldberg, D., Taylor, A., 2012. Risk of transmission associated with sharing drug injecting paraphernalia: An analysis of recent hepatitis $\mathrm{C}$ infection using cross-sectional survey data. Unpublished Manuscript. 
Pouget, E.R., Hagan, H., des Jarlais, D.C., 2012. Meta-analysis of hepatitis C seroconversion in relation to shared syringes and drug preparation equipment. Addiction 107, 1057-1065.

Roy, E., Alary, M., Morissette, C., Leclerc, P., Boudreau, J.F., Parent, R., Rochefort, J., Caessens, C., The SurvUDI Working Group, 2007. High hepatitis C virus prevalence and incidence among Canadian intravenous drug users. Int. J. STD AIDS 18, 23-27.

Scottish Government, 2008. Hepatitis C action plan for Scotland. Phase II: May 2008-March 2011. http://www.scotland.gov.uk/Publications/2008/05/ 13103055/0. Accessed (2011). Edinburgh, UK.

Scottish Government, 2010. Guidelines for services providing injecting equipment. Best practice recommendations for commissioners and injecting equipment provision (IEP) services in Scotland. http://www . scotland.gov.uk/Publications/ 2010/03/29165055/0. Accessed (2011). Edinburgh, UK.

Simmonds, P., Mutimer, D., Follett, E., 1998. Microbiology and microbial infections. Arnold, London, UK. chapter 35.

Speed, S., 1998. The sharing of injecting paraphernalia among 96 regular attenders at needle exchange schemes in the North West of England: Implications for local public health policy. Int. J. Drug Policy 9, 351-358.

Sutton, A.J., Gay, N.J., Edmunds, W.J., Hope, V.D., Gill, O.N., Hickman, M., 2006. Modelling the force of infection for hepatitis $\mathrm{B}$ and hepatitis $\mathrm{C}$ in injecting drug users in England and Wales. B.M.C. Infect. Dis. 6, 1-10.

The Kirby Institute, 2011. Austalian NSP survey: Prevalence of HIV, HCV and injecting and sexual behaviour among NSP attendees. National Data Report 1995-2010. Technical Report. The Kirby Institute for Infection and Immunity in Society, University of New South Wales, Sidney, Australia.

Thibault, V., Bara, J.L., Nefau, T., Garson, C.D., 2011. Hepatitis C transmission in injection drug users: Could swabs be the main culprit? J. Infect. Dis. 12, 18391842.

Thorpe, L.E., Ouellet, L.J., Hershow, R., Bailey, S.L., Williams, I.T., Williamson, J., Monterroso, E.R., Garfein, R.S., 2002. Risk of hepatitis C virus infection among young adult injection drug users who share injection equipment. Am. J. Epidemiol. $155,645-653$.

Vickerman, P., Grebely, J., Dore, G.J., Sacks-Davis, R., Page, K., Thomas, D.L., Osburn, W.O., Cox, A.L., Aitken, C.K., Hickman, M., Hellard, M., 2012. The more you look, the more you find: Effects of hepatitis $\mathrm{C}$ virus testing interval on reinfection incidence and clearance and implications for future vaccine study design. J. Infect. Dis 205, 1342-1350. 
Vickerman, P., Hickman, M., Judd, A., 2007. Modelling the impact on hepatitis C transmission of reducing syringe sharing: London case study. Int. J. Epidemiol. 36, 396-405.

Vickerman, P., Platt, L., Hawkes, S., 2009. Modelling the transmission of HIV and $\mathrm{HCV}$ among injecting drug users in Rawalpindi, a low HCV prevalence setting in Pakistan. Sex. Transm. Infect. 85, 23-30. 